La

Révolution

française

\section{La Révolution française}

Cahiers de l'Institut d'histoire de la Révolution française

1 | 2012

L'attentat, objet d'histoire

\title{
Le moment terroriste de la chouannerie : des atteintes à l'ordre public aux attentats contre le Premier Consul
}

The terrorist moment of Chouannerie: from threats to public order to political attacks against the First Consul

\section{Aurélien Lignereux}

\section{OpenEdition}

Journals

Édition électronique

URL : http://journals.openedition.org//rf/390

DOI : $10.4000 / / r f .390$

ISSN : $2105-2557$

Éditeur

IHMC - Institut d'histoire moderne et contemporaine (UMR 8066)

Référence électronique

Aurélien Lignereux, «Le moment terroriste de la chouannerie : des atteintes à l'ordre public aux

attentats contre le Premier Consul », La Révolution française [En ligne], 1 | 2012, mis en ligne le 18 mars 2012, consulté le 19 avril 2019. URL : http://journals.openedition.org/Irf/390 ; DOI : 10.4000/Irf.390

Ce document a été généré automatiquement le 19 avril 2019.

(c) La Révolution française 


\title{
Le moment terroriste de la chouannerie : des atteintes à l'ordre public aux attentats contre le Premier Consul
}

\author{
The terrorist moment of Chouannerie: from threats to public order to political \\ attacks against the First Consul
}

\section{Aurélien Lignereux}

1 Il serait sans doute risqué de qualifier l'explosion du 3 Nivôse an 9 (24 décembre 1800), de "crime sans exemple dans l'histoire», selon les mots de Bonaparte, ou de "forfait inouï ", à la façon de Fouché ${ }^{1}$, pour en faire sinon le premier des attentats, du moins la première des voitures piégées. C'est pourtant ainsi que la Machine infernale a été ressentie par l'opinion ou du moins qu'elle lui a été présentée; ces comptes rendus officiels portent en eux d'autres préfigurations, celles de 1 a dénonciation de terroristes à la solde de l'étranger et de l'exploitation du choc terroriste au service de mesures d'exception. De telles projections ont leur part d'anachronisme. Inversement, il est réducteur de s'en tenir au constat d'un assassinat ciblé, qui aurait viré à l'attentat aveugle. Quoi qu'il en soit, questionner, comme y incitent de récentes relectures, la portée de cet événement, en aval comme en amont ${ }^{2}$, dans le temps long d'une histoire de la violence terroriste et de ses représentations, est une manière de dépasser les seuls acquis de l'historiographie napoléonienne, qui compte la Machine infernale ou la conspiration de l'an 12 au nombre de ses sujets favoris. Cet engorgement tient à la dimension sensationnelle des faits, comme tout ce qui touche à la vie de Napoléon et à la fascination pour la suite de hasards qui l'ont sauvé, à deux secondes ou à deux minutes près - les chronomètres des chroniqueurs divergent -, ou bien à l'inscription de ces actes dans le genre à succès des complots, enquêtes et procès célèbres ${ }^{3}$, ou encore à la distribution exceptionnelle, avec Napoléon, Fouché et Cadoudal pour premiers rôles. N'insistons pas : il suffit de dire que ces épisodes ont déjà été racontés sous toutes leurs 
coutures, sur tous les tons et sous tous les angles, de quoi inspirer versions romancées, émissions de radio et même téléfilm.

Ces approches ont leur légitimité, ne serait-ce que par leur popularité, mais elles concentrent nombre des défauts reprochés à l'histoire napoléonienne, tant sur la forme (récit) que sur le fond, en privilégiant d'illustres comploteurs ${ }^{4}$, incitant en réaction à des recherches pionnières sur les opposants plus ordinaires ${ }^{5}$. Or il y a lieu de réintégrer l'événement spectaculaire dans le tissu factuel qui lui donne sa portée et son intelligibilité. En l'occurrence, il s'agit de s'interroger sur la définition de l'attentat et sur son insertion dans le répertoire des chouans, afin de penser leur usage de la terreur. Il est possible en effet que la chouannerie ait momentanément mis en œuvre une stratégie associant activisme politique, clandestinité, violence publique et symbolique, autorisant dès lors à parler de terrorisme $e^{6}$. Pouvant bénéficier d'appels d'air historiographiques, à l'exemple des soldats perdus de George Mosse ${ }^{7}$, cette approche permet de sortir du schéma usuel qui dégrade les chouans en brigands dès lors qu'ils ne se plient pas à la pacification de l'hiver 1800. Cette lecture sommaire oublie que cette sortie de guerre est aussi une entrée en politique ratée, impossible même vu que la pacification selon Bonaparte équivaut à une dépolitisation, n'offrant aux opposants irréductibles que le registre terroriste pour exister politiquement. Aussi conviendrait-il d'enrichir l'éclairante typologie de Roger Dupuy sur les formes de la chouannerie d'une sixième séquence, pour préciser la catégorie élastique de chouannerie-brigandage ${ }^{8}$. Celle-ci rend fidèlement compte de la dynamique de survie de groupes acculés dans un environnement de plus en plus hostile, mais leurs violences ne sauraient se limiter à cet engrenage : elles ont aussi une intentionnalité politique, qui est de défaire la pacification sur le terrain et ainsi défier l'œuvre du Consulat. En parlant de la conversion de Cadoudal "à l'action terroriste ", Roger Dupuy reconnaît d'ailleurs que la phase du brigandage n'est ni statique ni univoque; l'objet du présent article sera de suggérer que cette stratégie terroriste chouanne s'inscrit dans la durée, au-delà de 1800-1804.

Travailler sur des complots implique cependant d'accepter la règle du genre, c'est-à-dire de se résigner à ne pas tout connaître et à se méfier de sources piégées. Au nombre des difficultés, signalons d'abord l'illusion rétrospective. L'écho d'un attentat engendre un double effet de sources ${ }^{10}$ : d'une part, les témoignages postérieurs se multiplient pour incriminer des terroristes jacobins, tapis dans l'ombre, mais qui se seraient trahis par quelque menace ou vantardise, que le dénonciateur croit se rappeler grâce au déclic produit par l'explosion; d'autre part, la police redécouvre des rapports antérieurs à l'attentat et qui, à sa lumière, font figure de signes annonciateurs. Le lieutenant de Beaupréau annonçait ainsi la veille de l'attentat que «les anciens chouans levaient une tête audacieuse, qu'ils débitaient avec profusion que le Premier Consul était assassiné et que la guerre chouannique allait recommencer ${ }^{11}$. Ce genre de mise en garde n'acquiert de l'importance que rétrospectivement: les archives fourmillent d'alarmes démenties pour la plupart par le cours ultérieur des événements. À plus forte raison, la relecture de la correspondance des maires de la Sarthe, trois semaines après l'explosion, vise à étayer la thèse d'un complot, en vue de justifier l'arrestation de Bourmont et de 78 anciens chouans ${ }^{12}$. Encore ne s'agit-il là que de sources dérivées. Le soupçon doit porter sur le cœur même des dossiers, dossiers constitués par des polices évidemment intéressées à l'affaire. L'intoxication policière constitue en effet un second écueil. Les archives sont émaillées de faux ou de propos falsifiés, d'agents infiltrés ou de chouans retournés. Cette suspicion a conduit nombre d'historiens à réduire la plupart des projets d'attentat à des 
provocations policières ${ }^{13}$. Et si la Police générale n'est pas en cause, il est possible de relire la conspiration de Cadoudal-Pichegru-Moreau comme le coup de maître du contreespionnage, pour se débarrasser des principaux opposants à Bonaparte ${ }^{14}$.

\section{Du terrorisme à la chouannerie}

4 Plutôt que de suivre un peu paresseusement le double processus de radicalisation et de marginalisation, qui conduit de la chouannerie au terrorisme, on adoptera une démarche rétrospective afin d'identifier la composante terroriste que porte en elle la chouannerie.

\section{Un air plein de poignards}

5 Il n'est pas question non plus de livrer une énième reconstitution des affaires de la Machine infernale et de la conspiration de l'an 12; mieux vaut remettre en contexte ce climat du Consulat que Fouché a su caractériser d'un mot fameux : "L'air est plein de poignards $\aleph^{15}$. Le Directoire fournit d'ailleurs quelques précédents, dont le projet exprimé par d'André, en mai 1798, de faire assassiner les directeurs, ainsi que les principales tentatives d'assassinat de Hoche. La première est opérée en avril 1796, sur la route de Loudéac, par un chef royaliste abandonné par ses hommes, qui a tenté d'abattre le général d'un coup de fusil, acceptant de se sacrifier dans la mesure où Lantivy du Rest était certain d'être taillé en pièces, quelle que soit l'issue ${ }^{16}$. La seconde, commanditée par Teyssière, a eu lieu le 15 octobre 1796, à Rennes, à la sortie du théâtre, mais les deux coups de pistolet tirés par Moriau, un forgeron au nom chouan de « Frappe-à-mort », ne font que toucher un mur de la place Saint-Melaine. Il n'est pas anodin que le bruit de la venue à Paris de Moriau a circulé en mai 1801, au sein d'un commando venu abattre Bonaparte ${ }^{17}$. Le tableau suivant résume les différents attentats projetés sous le Consulat.

Projets terroristes imputés aux royalistes sous le Consulat

\begin{tabular}{|c|c|c|}
\hline Date & Nom et protagonistes & Projet \\
\hline $\begin{array}{l}\text { Février-début } \\
\text { mai } 1800\end{array}$ & $\begin{array}{l}\text { "Conspiration anglaise" } \\
\text { Agence royaliste de Paris } \\
\text { (Hyde de Neuville, } \\
\text { Dupérou) }{ }^{18}\end{array}$ & $\begin{array}{l}\text { Coordination entre: } \\
\text { un soulèvement dans l'Ouest sous le } \\
\text { commandement d'un prince } \\
\text { la livraison de Brest aux Anglais le coup essentiel } \\
\text { à Paris confié à Margadel: neutralisation de } \\
\text { Bonaparte (enlèvement ou assassinat) }\end{array}$ \\
\hline $\begin{array}{l}\text { Conçu à } \\
\text { partir de juin } \\
1800, \\
\text { organisé } \\
\text { d'octobre à } \\
\text { décembre, } \\
\text { exécuté le } 24\end{array}$ & $\begin{array}{l}\text { Attentat du } 3 \text { Nivôse an } 9 . \\
\text { Officiers de Cadoudal } \\
\text { (Saint-Réjeant, Limoëlan, } \\
\text { Carbon, Joyaux, La Haye } \\
\text { Saint-Hilaire) }\end{array}$ & $\begin{array}{l}\text { Reprise de la "Conspiration anglaise ", avec } \\
\text { variations (livraison de Belle-Ile) } \\
\text { Incertitudes sur les modalités de l'élimination de } \\
\text { Bonaparte: choix de l'explosion à l'initiative du } \\
\text { groupe (après avoir initialement projeté d'abattre } \\
\text { Bonaparte au spectacle à coups de carabine à } \\
\text { vent) }\end{array}$ \\
\hline
\end{tabular}




\begin{tabular}{|c|c|c|}
\hline Avril 1801 & $\begin{array}{l}\text { Projets tyrannicides de } \\
\text { fanatiques religieux }\end{array}$ & $\begin{array}{l}\text { Assassinat de Bonaparte par une femme lors } \\
\text { d'une audience ou coup de force sur la route de } \\
\text { La Malmaison }^{19}\end{array}$ \\
\hline Mai 1801 & $\begin{array}{l}\text { François Piogé (dit } \\
\text { Pitié), Bans- } \\
\text { (Barco), Francdeleur }\end{array}$ & Suspectés de préparer un assassinat \\
\hline Janvier 1803 & $\begin{array}{l}\text { Picot, Lebourgeois } \\
\text { (chouans installés à Rouen) }\end{array}$ & \\
\hline $\begin{array}{l}\text { Août } \\
1803-1804\end{array}$ & $\begin{array}{l}\text { Conspiration de l'an } 12 \\
\text { (Cadoudal et } 46 \text { chouans } \\
\text { réunis-Pichegru-Moreau) }\end{array}$ & $\begin{array}{l}\text { Vaste combinaison mise en œuvre à partir de } \\
\text { l'enlèvement de Bonaparte (assaut contre son } \\
\text { escorte) }\end{array}$ \\
\hline
\end{tabular}

6 Les continuités sont évidentes. L'exécution de la conspiration anglaise incombait au chevalier de Margadel, dit Joubert, officier émigré puis aide-major général de Frotté, ayant servi sous les ordres de Hingant de Saint-Maur et réputé pour ses actes de banditisme. Il avait réuni une douzaine d'hommes lorsque la conspiration fut découverte début mai 1800. L'attentat du 3 Nivôse s'y rattache doublement: c'est en juin que Cadoudal aurait formulé son projet, prenant le relais de la conspiration éventée ; l'envoi à Paris de Saint-Réjeant coïncide avec l'arrestation de Margadel, le 29 octobre, qui prive Hyde de tout moyen d'action. L'échec du 3 Nivôse n'éteint pas les menaces. En mai 1801, la police appréhende à Paris trois chouans de Cadoudal : Piogé, Burban et Francdeleur. En vérité, l'affaire est peu consistante: il n'y aura d'ailleurs pas de procès, mais Piogé, incriminé en raison de son passé et d'un prétendu repérage devant la Grande grille du Carrousel, restera treize ans durant prisonnier d'État ${ }^{20}$. En janvier 1803, deux autres assassins en puissance, aux ordres de Cadoudal, sont arrêtés à Paris: Picot et Lebourgeois ; ils sont exécutés le 27 janvier 1804.

\section{Aux sources du virage terroriste}

7 Cet état des lieux invite à réexaminer les sources supposées de la conversion terroriste. La spirale de la violence est une piste souvent suivie sur la foi du parcours des protagonistes de la Machine infernale, acculés à un dernier palier dans l'escalade. Cette radicalisation trahit la marginalisation de la chouannerie, abandonnée par les paysans et le clergé, poursuivant à Paris un combat désespéré. Bref, il s'agirait là d'un va-tout, résumant l'impasse criminelle d'une poignée d'enfants perdus, aveuglés par la haine, ne reculant pas devant des actes qui seront jugés odieux, mais prêts au contraire à en exploiter l'horreur pour compenser leur faiblesse. Cette présentation a du vrai ; cet isolement a d'ailleurs du bon. Passées les maladresses initiales et les contacts avec Hyde de Neuville et Coster Saint-Victor, le petit groupe a pu conduire son entreprise jusqu'à son terme parce qu'il est resté étranger aux réseaux royalistes de Paris, infiltrés par la police.

8 Un autre pan de l'historiographie minore la piste psychologique et raccorde l'événement à son contexte, ne serait-ce qu'au service d'une histoire-tableau ${ }^{21}$. L'élimination du Premier Consul serait dictée par l'évolution de la situation, résumée par l'impossibilité d'une reprise de la guerre à l'Ouest ${ }^{22}$ et par l'incarnation du Nouveau Régime en la 
personne de Bonaparte. L'attention à la chronologie et aux échelles emboîtées de l'Europe, de la France et de la Bretagne, pousse à faire du projet d'attentat le résultat de l'adaptation de Cadoudal aux opportunités et aux déconvenues d'une période qui va du départ de Bonaparte pour la deuxième campagne d'Italie jusqu'au traité de Lunéville. Cadoudal aurait été contraint de coller à l'événement, les plans plus ambitieux ayant été ruinés par Marengo (14 juin 1800) puis par Hohenlinden (3 décembre), avant de renoncer à toute entreprise à la suite du renversement de Pitt, le 8 février 1801. Le diagnostic politico-diplomatique a joué à plus forte raison pour la conspiration de l'an 12. Cette démarche rationnelle est d'autant plus volontiers prêtée aux royalistes qu'elle s'accorde avec les projets parallèles des exclusifs et enragés, acculés à la même radicalité.

L'historiographie blanche a d'ailleurs essayé de retirer aux royalistes la paternité du projet d'attentat en la réduisant à un emprunt momentané aux méthodes des conspirateurs jacobins. Des contemporains ont même affirmé que jacobins et chouans étaient de mèche, si l'on veut bien accepter ce jeu de mots. Fouché a en tout cas défendu la thèse d'une captation. Dans son rapport du 31 janvier 1801, il suppose qu' « [i]l y a apparence que l'idée du complot de Chevalier fit songer à l'explosion des barils de poudre. Cette idée aura été d'autant mieux accueillie que le soupçon du crime devoit naturellement tomber sur les auteurs du premier complot $»^{23}$. Elle est probable - vu la notoriété du potentiel de destruction de la Machine infernale de Chevalier ${ }^{24}-$, mais invérifiable - la police ayant perdu la trace du groupe chouan dans les semaines décisives qui ont précédé l'attentat et Saint-Réjeant s'étant muré dans le silence durant son procès. La confrontation de la liste suivante avec le précédent tableau des projets royalistes va dans le sens du mimétisme terroriste; ajoutons que la Police s'est emparée, dès le 4 Nivôse, de quatorze jacobins jugés capables de commettre un crime semblable et réputés avoir évoqué la possibilité de son exécution.

Projets terroristes imputés aux jacobins (juillet-décembre 1800) ${ }^{25}$

\begin{tabular}{|c|c|c|}
\hline Date & Nom et protagonistes & Projet \\
\hline & Jouvenot & $\begin{array}{l}\text { Enlever le Premier Consul sur la route de } \\
\text { Malmaison, ou l'assassiner dans son château } \\
\text { après avoir provoqué une panique en } \\
\text { incendiant une ferme voisine }\end{array}$ \\
\hline \multirow[t]{3}{*}{$\begin{array}{l}14 \text { juillet } \\
1800\end{array}$} & $\begin{array}{l}\text { Des soldats républicains } \\
\text { mécontents }\end{array}$ & Abattre Bonaparte à l'occasion de la revue \\
\hline & Le « nouveau Brutus» & Poignarder Bonaparte au spectacle \\
\hline & $\begin{array}{l}\text { Chapelle, Guibert, Dufour, Feon } \\
\text { et Rossignol } \\
\text { et autres jacobins installés dans } \\
\text { des cabarets de la rive droite }\end{array}$ & $\begin{array}{l}\text { Créer un tumulte à la porte d'un spectacle } \\
\text { auquel se trouverait le Premier Consul et le } \\
\text { poignarder à la faveur de ce mouvement de } \\
\text { foule. } \\
\text { Plan de repli : attaque de la voiture du Premier } \\
\text { Consul sur la route de Malmaison par } 30 \\
\text { hommes, bien déterminés et armés } \\
\text { d'espingoles }\end{array}$ \\
\hline
\end{tabular}




\begin{tabular}{|l|l|l|}
\hline 10 octobre & $\begin{array}{l}\text { Conspiration des poignards } \\
\text { (Aréna, Demerville, Diana, } \\
\text { Topino-Lebrun, Ceracchi.. et } \\
\text { Harel) }\end{array}$ & $\begin{array}{l}\text { Projet d'assassinat de Bonaparte au Théâtre } \\
\text { des Arts }\end{array}$ \\
\hline Conciliabules dans des cabarets & $\begin{array}{l}\text { Viser Bonaparte au sein même des Tuileries : } \\
\text { jeter des matières inflammables dans les cuves } \\
\text { du palais } \\
\text { s'introduire sous le château grâce à une espèce } \\
\text { d'aqueduc donnant sur la Seine } \\
\text { jeter des fusées }\end{array}$ \\
\hline $\begin{array}{l}\text { 3-8 } \\
\text { novembre } \\
1800\end{array}$ & Chevalier et Veycer & $\begin{array}{l}\text { Essai d'une machine infernale (baril de poudre, } \\
\text { de ferraille et de mitraille, monté sur un } \\
\text { cabriolet) }\end{array}$ \\
\hline
\end{tabular}

Enfin, une quatrième piste, celle d'un terrorisme en réplique à la terreur d'État, est à l'honneur dans l'historiographie royaliste: les attentats chouans sont des actes de légitime défense en réponse à une répression féroce, terroriste même. Cet enchaînement vindicatif est l'un des ressorts des guerres de l'Ouest; après le 18 Brumaire, il a pu servir de justification aux tentatives contre Bonaparte, à l'instar de l'exécution, le 25 janvier 1800, du chevalier de Toustain, un jeune officier de l'armée du Maine venu imprudemment se compromettre à Paris par l'achat de poignards et de plumets blancs. Sa mort provoque la fureur de Hyde de Neuville, qui écrit au comte d'Artois : «Ce sang crie vengeance; il la rend sainte et légitime. Hier, Félix, comme membre du gouvernement, n'avait pas l'odieux de l'assassinat; on pouvait balancer à le frapper; aujourd'hui je mettrais ma gloire à le poignarder $\aleph^{26}$. L'exécution de Louis de Frotté a été dictée par la raison d'État, pour donner un signe exemplaire de la résolution du pouvoir, avec le risque cependant de raidir les insoumis. De même, la traque sans merci dirigée contre Cadoudal a souvent été invoquée; citons pour mémoire ce billet de Bonaparte à Brune, du 3 juin 1800 : « Prenez mort ou vif ce coquin de Georges. Si vous le tenez, faites-le fusiller sur-lechamp comme ayant été en Angleterre après la pacification $»^{27}$. L'exécution déguisée de Julien Cadoudal, le frère de Georges, le 8 février 1801, s'inscrit dans de ce cycle, qui, outre l'armée et la police ${ }^{28}$, mobilise des chouans retournés mandatés pour assassiner Cadoudal. Ces tentatives avérées autorisent à parler de contre-terrorisme, soit l'utilisation de moyens analogues à ceux des terroristes pour lutter contre eux. Enfin, la montée en puissance des enlèvements dans le répertoire chouan après 1799 peut être considérée comme une riposte à la loi des otages. Bien qu'abolie, elle aurait encouragé des répliques similaires. D'ailleurs, là encore, les cloisons ne sont pas étanches: début 1803, Bonaparte faisait étudier la possibilité d'enlever le comte d'Antraigues, installé en Saxe, et on connaît le sort du duc d'Enghien. L'enlèvement du Premier Consul, au cœur de la conspiration de l'an 12, doit être replacé dans ce cadre.

\section{L'usage de la terreur au cœur de la chouannerie}

11 Cette mise en contexte doit être prolongée par une mise en perspective interrogeant l'usage de la terreur par la chouannerie. Toute la question est de savoir si cette terreur semée dans l'Ouest est l'objet d'une stratégie délibérée, autrement dit si elle relève du 
terrorisme. Articulée autour des guerres (1794, 1795-1796, été 1799-hiver 1800), la chronologie de la chouannerie dessine en creux des phases marquées par des violences ponctuelles que les traités ne parviennent pas à juguler. C'est dans ces intervalles que se nichent les actions de type terroriste. En 1798, face au raidissement néo-jacobin et pour contourner l'interdiction par Louis [XVIII] d'une reprise d'armes, les chefs insoumis ont multiplié les coups de main afin de conserver le contrôle de leurs hommes et du pays. La coïncidence de cet entre-chouannage ou chouannerie d'officiers avec la vague nationale de grand brigandage a conforté les autorités républicaines dans leur conviction d'être confrontées à un plan concerté, comme l'exprime cette harangue du 29 novembre 1798 :

«Un système suivi de vols et d'assassinats semble s'organiser dans toute la République ; depuis longtemps il se fait sentir dans l'étendue de notre département. Des restes d'émigrés et de brigands armés, joints à une bande de scélérats échappés des fers, parcourent les campagnes et y répandent la terreur et l'effroi [...]. Je ne vous rappellerai point, citoyens, les attentats commis depuis un an dans les communes de [quatorze localités], etc... Tantôt, c'est une voiture publique enlevée, la correspondance des autorités interceptées, les fonds de la nation volés ; tantôt c'est une ferme dévastée, un père de famille ruiné ; ici, un fonctionnaire public tombe sous le fer des assassins ; une famille entière succombe sous les douleurs cuisantes ; [...] le vol et l'assassinat deviennent aujourd'hui, dans les mains de nos ennemis, les deux principaux ressorts de la contrerévolution... $»^{29}$.

Une même emprise terroriste pèserait sur le Morbihan, du moins sous la plume experte de Montgaillard: «Entre les deux guerres, Georges se tint toujours caché dans son pays, et en relation avec l'Angleterre. Le Morbihan paraissait tranquille, et cependant une nouvelle insurrection y était un feu caché que le chef pouvait découvrir à volonté. Ses moyens étaient la terreur qu'il entretenait par des exécutions fréquentes, confiées à un certain nombre de forcenés à sa solde [...] $»^{30}$. Cet état de 1798 sert à créer un parallèle avec 1800 , pour ainsi étayer dans la durée cette stratégie terroriste. De fait, l'autre phase de terreur chouanne se déroule de juin 1800 à janvier 1801, sous le nom de chouannerie à volonté et sur un mode mineur, en raison de la passivité des populations et des prêtres satisfaits de la paix offerte par le Consulat, et du rapport de forces hérité de Marengo. Dans ces conditions, Cadoudal autorise les initiatives ponctuelles, destinées à défier localement l'ordre bonapartiste.

Il y a donc lieu de revenir sur le sens d'actions qui, prises une à une, relèvent du brigandage, mais dont l'écho, sciemment escompté, confère une dimension terroriste. Tel est le cas de l'« horrible attentat » contre le gendarme Michelet, enlevé de son auberge de Jean-sur-Erve, au su et même au vu des habitants, la nuit du 8 janvier 1799, et fusillé un peu plus loin ${ }^{31}$. Ce type d'actions met en lumière la fragilité des patriotes, abandonnés par les populations, et oblige les autorités à user de la responsabilité communale, au risque de conforter l'hostilité des habitants. Là où les chouans ne sont pas en force, une pancarte sur le corps des victimes revendique le châtiment des traîtres pour dissuader toute collaboration. Dans des conjonctures désespérées, des commandos peuvent répandre la panique et l'anarchie par un raid terrible, comme la bande de Boisguy à Antrain et SaintOuen-la-Rouërie dans la nuit du 7-8 avril 1796. Le 15 janvier 1800, à Sap (Orne), huit membres de l'administration municipale du canton auraient été massacrés, à la hache semble-t-il, et le juge de paix brûlé vif sur la place. Horreur payante puisque le contrôle de ce canton républicain est en passe d'être perdu sous l'effet de la terreur: "Tous les citoyens fuient devant des tigres aussi cruels, l'administration centrale sera bientôt la 
seule existante dans ce département $»^{32}$. De fait, l'exode des fonctionnaires et des patriotes notoires prive le commissaire central de toute correspondance dans des circonstances où elle est plus nécessaire que jamais, et le réduit à réclamer des troupes pour mettre fin aux « attentats » de ces « cannibales »33. Il n'est pas anodin que Picot, l'un des deux hommes envoyés à Paris en janvier 1803 par Cadoudal, était compromis avec sa bande dans le massacre de Sap.

\section{La chouannerie à volonté et l'attentat du 3 Nivôse}

L'attentat du 3 Nivôse participe à plusieurs titres de la «chouannerie à volonté ". Premièrement, la mission initiale de Saint-Réjeant était de s'emparer de Bonaparte, clôturant une série de guet-apens spectaculaires en 1800: outre l'échec de tentatives contre les préfets du Finistère et du Morbihan, l'enlèvement du sénateur Clément de Ris, en septembre, opéré par d'anciens officiers de Bourmont et de Frotté, ou, en novembre, l'assassinat d'Audrein, évêque du Finistère, arraché de sa diligence et abattu par François Le Cat, jeune officier dans la $5^{\mathrm{e}}$ légion de Cadoudal. S'appuyant sur les révélations de l'indicateur Desgrées mentionnant une lettre de Cadoudal à Saint-Réjeant, Fouché fera de ce crime l'aiguillon du 3 Nivôse ${ }^{34}$. Un enlèvement dans la lande bretonne est cependant plus aisé à réaliser qu'aux abords de la capitale ; Saint-Réjeant a vite constaté la vanité du projet, avec la poignée d'hommes dont il disposait. En 1803, Cadoudal a néanmoins cru l'entreprise possible et s'en est donné les moyens : 46 hommes d'action auront été réunis à Paris. Deuxièmement, il est à souligner que la deuxième quinzaine de décembre, avec une dizaine d'exécutions contre des dénonciateurs ou des fonctionnaires, marque le pic d'un «système d'assassinats partiels $»^{35}$; le meurtre du Premier Consul en aurait été le point d'orgue. Troisièmement, Joyaux, La Haye Saint-Hilaire, Limoëlan et Carbon ont d'abord, à la mi-Brumaire, transposé la chouannerie-brigandage aux environs de la capitale. D'après Du Châtellier, un traître employé par Desmarest, les chouans opéraient des repérages sur la route de Charenton, en vue d'intercepter la diligence de Troyes. C'est l'arrivée d'un nouvel agent de Georges, Saint-Réjeant, qui a réorienté le groupe. Quatrièmement, les antécédents des terroristes les ancrent dans la chouannerie à volonté.

Itinéraire des chouans impliqués dans l'attentat du 3 Nivôse

\begin{tabular}{|c|c|c|c|}
\hline Noms & $\begin{array}{l}\text { Origines } \\
\text { formation }\end{array}$ & Passé dans la chouannerie & Sort \\
\hline $\begin{array}{l}\text { Pierre Robinault } \\
\text { de Saint-Réjeant } \\
\text { ou Saint-Régent } \\
\text { dit Pierrot }\end{array}$ & 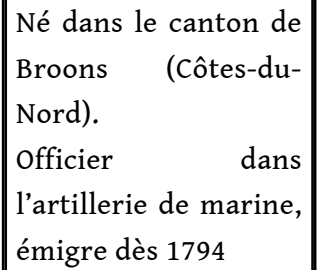 & $\begin{array}{l}\text { A servi sous Puisaye, présent à } \\
\text { Quiberon, blessé à Locminé (1796). } \\
\text { Passe en Angleterre avec Cadoudal } \\
\text { (1798). Commande la légion de la } \\
\text { Trinité-Porhoët, } 1799\end{array}$ & $\begin{array}{l}\text { Guillotiné le } \\
20 \text { avril } 1801\end{array}$ \\
\hline
\end{tabular}




\begin{tabular}{|c|c|c|c|}
\hline $\begin{array}{l}\text { Joseph Picot de } \\
\text { Limoëlan } \\
\text { dit Pourleroy, puis } \\
\text { Beaumont }\end{array}$ & 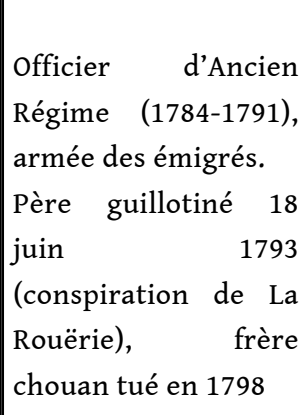 & $\begin{array}{l}\text { Entré en chouannerie en } 1799 . \\
\text { Chargé du district de Broons où il } \\
\text { cherche à mener une guerre } \\
\text { convenable, même s'il s'en remet } \\
\text { parfois à des hommes de terrain, en } \\
\text { lisière du brigandage, comme les } \\
\text { frères Bobon }\end{array}$ & $\begin{array}{l}\text { Fuite aux } \\
\text { États-Unis, } \\
\text { prêtrise en } \\
1812\end{array}$ \\
\hline $\begin{array}{l}\text { François-Joseph } \\
\text { ou Jean Carbon } \\
\text { dit le Petit- } \\
\text { François }\end{array}$ & $\begin{array}{l}\text { Parisien de } \\
\text { naissance, chirurgien } \\
\text { de marine, présenté } \\
\text { comme un voleur } \\
\text { d'église }\end{array}$ & $\begin{array}{l}\text { Chouannerie en Ille-et-Vilaine, sous } \\
\text { les ordres d'Achille Le Blond }\end{array}$ & $\begin{array}{l}\text { Guillotiné le } \\
20 \text { avril } 1801\end{array}$ \\
\hline $\begin{array}{l}\text { Armand-Aimé } \\
\text { Joyaux dit dit } \\
\text { d'Assas } \\
\text { Villeneuve }\end{array}$ & $\begin{array}{l}\text { Famille aisée, mais } \\
\text { présenté comme un } \\
\text { débauché, } \\
\text { habitudes } \\
\text { spadassin }\end{array}$ & $\begin{array}{l}\text { Aide de camp de Georges, } \\
\text { conspiration de l'an } 12\end{array}$ & $\begin{array}{l}\text { Guillotiné le } \\
12 \text { juin } 1804\end{array}$ \\
\hline $\begin{array}{l}\text { Édouard de La } \\
\text { Haye Saint- } \\
\text { Hilaire } \\
\text { dit Dieudonné }\end{array}$ & $\begin{array}{l}\text { Né à } \text { St-Hilaire-des- } \\
\text { Landes } \quad \text { (Ille-et- } \\
\text { Vilaine) }\end{array}$ & $\begin{array}{l}\text { Armée de Cadoudal (Pont-du-Loc), } \\
\text { conspiration de l'an 12, enlèvements, } \\
\text { dont celui de l'évêque de Vannes }\end{array}$ & $\begin{array}{l}\text { Exécuté le } 7 \\
\text { octobre } 1807\end{array}$ \\
\hline
\end{tabular}

Au-delà des itinéraires, la peinture des caractères est exemplaire. Saint-Réjeant retient l'attention, non seulement en raison de sa fonction (il est « chargé particulièrement de l'exécution militaire de tous les ordres qui arrivent à Paris $»^{36}$ ), mais aussi de son expérience (officier dans l'artillerie de marine, il a la compétence d'artificier nécessaire pour la Machine infernale) et de sa réputation ambivalente dans les milieux chouans, celle d'un homme dévoué et impitoyable, brave jusqu'à la témérité, accrédité par ses faits d'armes: pillage de la caisse de Loudéac, au domicile même du receveur (13 novembre 1798), attaques d'une diligence entre Ploërmel et Plélan-le-Grand (février 1799) puis de la caisse de Loudéac, au cours de laquelle quatre membres de l'escorte furent tués (26 juillet 1799), participation au raid sur Saint-Brieuc (26 octobre 1799) ; avant de partir à Paris, il chouannait à volonté dans les Côtes-du-Nord. Il était passé maître dans l'art de la dissimulation, campant dans une loge de terre, de mousse et de branchages, en forêt de La Noué. On ajoute qu'il avait le vin mauvais : six cultivateurs de Ménéac en perdirent la vie. Le parcours de Limoëlan est moins flamboyant, aussi pour donner du relief à cet homme, chargé de l'intendance du groupe à Paris, la propagande en fera un esprit ténébreux et haineux. Carbon est présenté quant à lui comme l'archétype du chouan populaire, brutal et cruel. Les amnistiés le dépeindront comme « un scélérat de l'armée de Georges, qui a servi dans son artillerie, célèbre par ses assassinats, faisant métier de tuer des bleus $»^{37}$. Les autorités ont beau jeu d'en faire un criminel d'habitude, condamné en 1794 pour vol de linge dans l'église de Mantes et spécialisé dans les attaques de diligence. Joyaux fait figure de débauché prêt à tout ; il a participé à la Machine infernale, sans doute déguisé 
en charretier, mais est parvenu à quitter Paris avec La Haye Saint-Hilaire, lui aussi chouan éprouvé, partisan de l'assassinat de Bonaparte dès sa fameuse entrevue avec Cadoudal, le 5 mars 1800, aux Tuileries, et qui, de retour en Bretagne, opérera des enlèvements politico-crapuleux (Veillet-Dufrêche, de Tréveneuc), et qui sera comme Joyaux de la conspiration de l'an 12. De fait, celle-ci est pour moitié l'œuvre de chouans ; citons, outre Cadoudal, Picot dit le Bourreau des Bleus, Roger dit Loiseau, Ducorps, Hervé dit Sans façon, les frères Gaillard, Deville dit Tamerlan, Mérille dit Beauregard, Sol de Grisolles, Pierre-Jean Cadoudal, La Grimaudière dit Lallemand, Lelan dit Brutus, ou François Fouché.

\section{Du terrorisme à l'anti-terrorisme}

\section{Le terrorisme, un anachronisme?}

17 Avant même d'ajouter Paris à son terrain d'action, la chouannerie a une composante terroriste. Les citations précédentes prouvent du reste que le mot attentat, incontestablement associé à l'explosion de la rue Nicaise, s'emploie aussi pour qualifier tout acte de violence ayant des répercussions sur l'ordre public, tels que des crimes contre des particuliers mais forts d'une évidente portée politique dans l'Ouest. Le Code des délits et des peines du 3 Brumaire an 4 (25 octobre 1795) n'utilise qu'au sens large ce terme élastique : Saint-Réjeant et Carbon ont notamment été condamnés en vertu des articles 612, 611 et 613, prévoyant la mort contre les individus qui auront comploté « pour troubler la république par une guerre civile, en armant les citoyens les uns contre les autres, et contre l'exercice de l'autorité légitime», mais aussi qui auront commis «un homicide avec préméditation ", ce qui s'applique aux passants tués, et auront tenté un assassinat "quoique non consommé », ce qui vaut pour Bonaparte. L'usage de terrorisme est plus problématique, d'abord parce qu'il n'apparait jamais, ensuite parce que son dérivé terroriste est alors réservé aux jacobins, dans le souvenir revisité de la Terreur de 1793-1794. Enragés, exclusifs, anarchistes, septembriseurs: les termes sont pourtant nombreux pour désigner les hommes qui ne se résignent pas au mot d'ordre d'une Révolution terminée, mais celui de terroristes leur reste exclusif, d'autant plus que la poudre de Chevalier était en passe de fixer l'acception contemporaine du mot, en le faisant glisser d'une signification incantatoire à une définition opératoire. Le passage à l'acte des chouans n'a pas suffi à un partage du terme; les représentations antérieures restent dominantes : ils demeurent des brigands dans le discours officiel.

Un portrait type du terroriste ne s'en constitue pas moins alors, empruntant nombre de ses traits aux chouans. Rarement signalements n'auront été autant diffusés que ceux des suspects de la rue Nicaise et plus encore que ceux des conspirateurs de l'an 12 . Cependant, si ces affiches cherchent à mettre en garde l'opinion, elles se doivent surtout d'être fidèles; ainsi en est-il des 63 visages dessinés de profil ou de trois-quarts des Portraits exacts des Conspirateurs chargés par le Gouvernement britannique d'attenter aux Jours $d u 1^{\text {er }}$ Consul. Le signalement de Georges, publié dans le Moniteur du 27 février 1804 et affiché dans les rues de Paris, tend toutefois à en faire un monstre de foire: « extrêmement puissant et ventru, épaules larges, d'une corpulence énorme, la tête trèsremarquable par sa prodigieuse épaisseur, cou très court, le poignet fort, doigts courts et gros, jambes et cuisses pas très longues, le nez écrasé et comme coupé dans le haut, large du bas ». On comprend mieux pourquoi Cadoudal a pu vivre six mois à quelques centaines 
de mètres de la Préfecture de police et que les badauds, contrairement aux représentations complaisantes, se sont défilés lors de la course-poursuite de la Montagne sainte-Geneviève dès qu'ils ont su que l'individu coursé n'était pas un voleur mais celui que la presse présentait comme un tueur prêt à tout.

De fait, le fanatisme est au cœur des représentations et des raisons pour rendre compte de l'engagement terroriste. Simple cordonnier rennais, Hervé est jugé dangereux parce qu' " ancien chouan très fanatique $»^{38}$, Sol de Grisolles est dépeint en "homme très dangereux, fanatique, ennemi implacable et violent de toutes les nouvelles institutions ${ }^{39}$ , et Napoléon aurait dit de Cadoudal: «C'était un fanatique ; je l'émus sans parvenir à le convaincre $»^{40}$. Pareil fanatisme les rend irrécupérables pour la société comme d'ailleurs incontrôlables pour leurs alliés politiques. Les brochures officieuses ne se font pas faute de reprocher à Moreau son imprévoyance : "Moreau régnerait maintenant s'il n'était point déjà poignardé par les complices qu'il voulait rendre les instrumens de son ambition, et qui l'eussent sans doute sacrifié à leur fanatisme pour les Bourbons » ${ }^{41}$. La réconciliation nationale voulue par Bonaparte jette le soupçon sur les minorités, condamne les extrêmes et érige le fanatisme en repoussoir absolu. C'est pourquoi Montgaillard ne craint pas de donner quelque publicité aux propos attribués à Le Cat et à ses hommes, après l'assassinat de l'évêque Audrein, avertissant les autres voyageurs "qu'ils n'étaient pas des voleurs, mais des royalistes " $^{42}$. Les chouans ont cru justifier leur geste et sauver leur réputation, mais dans l'échelle des valeurs, tuer pour des idées ne vaut pas mieux qu'assassiner par lucre. Le Journal de Paris du 17 Germinal an 9 fait immédiatement suivre dans ses colonnes l'annonce de la condamnation à mort de Carbon et de Saint-Réjeant par le compte rendu d'un poème allégorique et philosophique de Louis Lemercier. Ces Trois fanatiques ridiculisent les meurtres mutuels projetés par «Trois insensés égarés par le fanatisme, caché sous le masque de religion, de patrie et de liberté ", avant d'exalter leur retour à la raison. L'explosion du 3 Nivôse voue aux gémonies l'extrémisme: l'absence de compromis exige non seulement l'élimination physique de l'adversaire mais accepte encore si nécessaire celle de tiers. Les comploteurs ont admis que la mort de Bonaparte justifiait un inévitable carnage à Paris, sans avoir bien mesuré le caractère aléatoire de l'entreprise, que Monge, appelé à se prononcer en expert par la préfecture sur la machine de Chevalier, avait mis en évidence ; il concluait " que si l'auteur est sensé, la machine ne paraît pas avoir été imaginée pour mettre à mort une personne déterminée, mais bien pour blesser et même tuer indistinctement une grande quantité de personnes réunies, comme, par exemple, dans un cas d'abordage ${ }^{43}$. Bref, les comploteurs ont employé pour un assassinat les moyens techniques de la violence terroriste, ouvrant une nouvelle ère de la violence politique ${ }^{44}$.

En partie involontaire, ce basculement n'a pas été assumé ; autrement dit, l'attentat n'a pas été revendiqué. Cela s'inscrit il est vrai dans la stratégie des comploteurs qui escomptaient que l'opinion imputerait le crime aux jacobins, un crime dont les dividendes seraient royalistes. Les chouans misaient en effet sur le vide politique créé par la mort de Bonaparte. Au fanatisme et au cynisme de Cadoudal, Fouché ajoute encore le machiavélisme pour lui attribuer, dans son rapport du 11 Pluviôse, les conjectures suivantes: «les jacobins auraient profité du désordre pour tenter de s'emparer du gouvernement. Alors plus de paix, les Autrichiens occupèrent nos troupes dans l'intérieur de l'Allemagne. La guerre se rétablissait facilement dans la Vendée, dans tout l'Ouest, en persuadant aux habitants qu'on les armait pour les soustraire à la domination des Terroristes, ennemis de la religion et de tout gouvernement modéré ${ }^{45}$. Cadoudal aurait 
parié sur la politique du pire pour remobiliser l'Ouest et attirer la France des honnêtes gens, à moins que le désarroi n'eût provoqué un ralliement à la monarchie, en un réflexe que l'arrivée opportune d'un prince aurait su susciter. Le volet politique sera soigné dans la conspiration de l'an 12 : une fois Bonaparte éliminé, le triumvirat Cadoudal, Pichegru et Moreau, ralliant à eux des pans complémentaires de l'opinion, assurera la transition monarchique.

Ces calculs supposent donc la fragilité d'une République suspendue à la vie de Bonaparte. En Nivôse an 9, la nature personnelle du pouvoir étant brutalement mise à nu, les autorités ont réagi dans une double direction : prévenir les émules de Saint-Réjeant que le régime était solide et saurait survivre au Premier consul ; rassurer l'opinion. Le message est relayé par la presse et par des feuilles occasionnelles, telles que la Clef du cabinet, qui, le 2 Pluviôse, garantit la solidité de la République consulaire, forte de l'appui des corps constitués, de l'armée et de l'opinion, ce qui la met à l'abri des «scélérats à bonnet ou talons rouges", thématique que reprend avec emphase l'exorde du commissaire du gouvernement, le 13 Germinal an 9, devant le tribunal criminel de la Seine. La ligne politique est du reste ambivalente dans la mesure où le jugement impose aux condamnés la chemise rouge des parricides. L'opinion n'en demeure pas moins inquiète, comme en témoignent certaines rumeurs : celle de l'empoisonnement de Bonaparte, en avril 1801, jette l'alarme dans le Rhône au point de contraindre le préfet à un démenti officiel ${ }^{46}$. En l'an 12, la menace consolidera le nouvel ordre, sous la forme d'un empire héréditaire, à en croire d'explicites adresses: "Ce vœu était dans tous les cœurs, mais il fallait une occasion pour le développer; l'affreuse conspiration de Georges, qui devait être si funeste à la République, a été comme l'étincelle électrique dont toutes nos âmes ont ressenti au même instant la commotion; et sur le champ l'Empire français s'est élevé sur une base désormais inébranlable $»^{47}$.

\section{La naissance de l'antiterrorisme}

Cette communication politique joue donc sur l'émotion publique. Les huit-neuf morts de la rue Nicaise - mais on a pu gonfler le bilan à vingt-deux tués - ont provoqué un mouvement de compassion et d'indignation au sein d'une opinion choquée par les circonstances du carnage à l'encontre de passants littéralement déchirés par cette explosion d'un baril de poudre et de ferraille. Le commissaire du gouvernement s'étendra sur les mutilations des victimes à grands renforts d'images puisées dans le registre frénétique devant le tribunal criminel, le 13 Germinal an 9. Aucun parti ne pouvait assumer cette mort de soldats réservée à d'innocents Parisiens. Dans les rangs royalistes, l'idée même d'un assassinat n'était pas avouable et ce n'est bien qu'à titre métaphorique que des poèmes pouvaient exalter l'idée d'attentat : «Jupiter prête nous ta foudre / Pour venger la cause du Roi / Nous jurons de réduire en poudre / Les législateurs et les loix [... ] $\aleph^{48}$, pour reprendre le texte d'une chanson saisie à Bayeux chez Mme Vaudabon, épouse d'un émigré, le 20 octobre 1800. Le passage à l'acte a été désavoué dans les milieux royalistes, et cela d'autant plus qu'il passait d'abord pour un coup des jacobins dont il portait l'infamie. Louis [XVIII] s'en est indigné ; emprisonné, Louis d'Andigné écrira qu'il abhorre cet attentat. Du côté des auteurs et des commanditaires, c'est la consternation : l'attentat n'avait pour seule justification que la mort de Bonaparte. Déjà le projet d'assassinat avait conduit Hyde de Neuville et Coster Saint-Victor à se retirer du complot, et Limoëlan répugnait aux modalités d'une machine infernale. Cadoudal enrage ; il paraît acquis qu'il a commandité l'action du groupe chouan, qu'il a accepté l'éventualité de la 
mort de Bonaparte, sans avoir conçu ni connu le projet d'une voiture piégée. Deux éléments sont certains : d'une part, les autorités se sont efforcées de lui attribuer la décision de "faire sauter ce fils de p... de Bonaparte », comme le rapporte le réquisitoire du commissaire du gouvernement, quitte à façonner des indices; d'autre part, Cadoudal a tiré la leçon de l'effet déplorable de l'attentat, se donnant les moyens de réussir l'enlèvement de Bonaparte, à la suite d'un assaut contre son escorte, en une espèce de nouveau combat des Trente. Les risques qu'une telle mobilisation comportait (réunion des hommes, repérages, confection d'uniformes) étaient le prix à payer pour obtenir l'aval du comte d'Artois et de la haute émigration, convaincus que leur retour ne pouvait s'opérer que par des voies honorables, sous peine de se mettre à dos l'opinion.

Le public a été frappé par l'expérience de la mort aveugle ${ }^{49}$. Le succès de la souscription en faveur des victimes indigentes et la teneur des adresses accompagnant les dons manifestent l'élan de sollicitude. Les autorités, qui ont su l'entretenir par la publicité accordée aux donateurs, n'ont pas manqué de faire un test politique de l'épreuve endurée, à l'instar du préfet de police célébrant « l'un hommage bien éclatant rendu à son génie, à ses vertus; ils sont la preuve la moins équivoque de l'amour sincère du peuple français $»^{50}$. La Police expérimente également le rôle d'un tel événement comme dérivatif des difficultés ordinaires. On rapporte ainsi que «la classe des petits marchands, des ouvriers qui se plaignait chaque jour du retard de la paix, de la stagnation du commerce, de la misère qui en résulte, paraît avoir oublié ses peines, pour ne s'occuper que du danger qui a menacé la société entière. [...] Entre divers propos: "Si nous pouvions les attraper, nous leurs ferions leurs fêtes [sic] ; ils seraient bientôt jugés" $»^{51}$. Le ressort de justice populaire peut s'avérer utile pour saisir les suspects. Inversement, faute de pouvoir jouer sur le choc d'un attentat, la campagne de presse de l'an 12 peine à mobiliser l'opinion, se heurtant même au scepticisme frondeur en faveur de Moreau. D'où le recours à des publicistes, s'efforçant de souligner l'ampleur de la menace : «Bien loin d'être ridicule, comme affecte de le dire le général Moreau, cette conspiration est la plus redoutable qui ait existé, non contre le Premier Consul qui a souvent échappé aux poignards et aux explosions préparés par quelques-uns des mêmes conspirateurs, mais contre l'État $\aleph^{52}$. La main de l'Angleterre doit contribuer à faire naître l'indignation ${ }^{53}$. L'enjeu est essentiel puisqu'il s'agit de faire admettre des mesures d'exception.

L'adage qui veut la fin veut les moyens vaut aussi bien pour le terrorisme que pour la lutte anti-terroriste; Sol de Grisolles, Picot, Bouvet de Lozier, Burban, Roger ou Coster Saint-Victor se plaindront d'avoir été torturés. Ces méthodes restent dissimulées. En revanche, l'émoi causé par l'attentat ouvre une opportunité politique rare, celle d'un consentement à une réplique énergique. La Police se plait à répercuter certains propos comme ceux qui auraient été entendus lors de la parade du 5 Nivôse : "L'indignation est si forte que de, toutes parts, on demande des exécutions exemplaires, sans observer les formes que la justice prescrit $»^{54}$. Les mesures d'exception tirent leur légitimité sinon d'une demande populaire, du moins de la mise en avant des devoirs fondamentaux de l'État envers les citoyens, menacés par une minorité. Le même jour, Bonaparte tient ce langage significatif aux maires des douze arrondissements : «Tant que cette poignée de brigands m'a attaqué directement, j'ai dû laisser aux lois et aux tribunaux ordinaires leur punition : mais puisqu'ils viennent, par un crime sans exemple dans l'histoire, de mettre en danger une partie de la population de la cité, la punition sera aussi prompte qu'exemplaire [...] je n'oublierai pas que mon premier devoir est de veiller à la défense du peuple contre ses ennemis intérieurs et extérieurs ${ }^{55}$. 

sentences impitoyables prononcées contre les pâles comploteurs jacobins de l'automne ni sur l'arrêté du 14 Nivôse an 12 proscrivant une centaine de républicains. L'arbitraire caractérise également l'arrestation de Bourmont et de 78 royalistes. Au-delà, le gouvernement profite des circonstances pour imposer aux Tribuns, le 7 février 1801, une loi instituant des tribunaux spéciaux, sans jury, ni appel, ni cassation. Le choc du 3 Nivôse motive même certains jugements : en juin 1801, le tribunal spécial de Maine-et-Loire condamne à mort neuf chouans amnistiés (sur dix-neuf accusés), sentence justifiée au prétexte que leurs vols et assassinats « préludaient à l'attentat du 3 Nivôse et coïncidaient avec lui. C'est le non succès de cet horrible attentat qui a neutralisé leurs brigandages $»^{56}$. Ce que les coups de main répétés des chouans n'avaient pas suffi à créer dans l'Ouest, malgré le climat de terreur ou du moins d'insécurité ainsi entretenu, la Machine infernale l'aura réalisé: l'attentat spectaculaire en contexte urbain fait événement, donne naissance au terrorisme comme usage calculé de la violence aveugle au service de buts politiques. Les ambiguïtés mêmes de ce « coup essentiel » contribuent à la postérité de ce type d'action, bien au-delà des combats chouans. Étroitement mêlé à l'affaire, Desmarest en fera la matrice d'une modernité politique, en tant qu'« expédition de commande, réglée en conseil de hauts personnages confiée aux mains d'hommes aussi adroits qu'intrépides. Leur plan et leurs préparatifs furent dressés de longue main, toutes les chances d'action calculées avec sang-froid et précision ${ }^{57}$. Son échec incite pourtant les chouans à revenir à un répertoire de déstabilisation plus familier, et les enlèvements perdureront même après le fiasco de la conspiration de l'an 12, à l'exemple de celui de l'évêque très concordataire de Vannes, le 23 août 1806, par La Haye Saint-Hilaire ${ }^{58}$, ou encore la découverte l'été suivant, d'un plan combiné par d'anciens complices de Cadoudal, prévoyant d'enlever Fouché, Moncey, Dubois, c'est-à-dire de paralyser la Police générale, la gendarmerie et la préfecture de Police, avant de s'emparer de Napoléon, le juger et l'exécuter ${ }^{59}$.

\section{NOTES}

1. Le Premier Consul aux maires et adjoints des douze arrondissements de Paris, 26 décembre 1800 (Journal de Paris, 7 Nivôse an 9) ; rapport du ministre de la Police générale concernant l'Attentat commis contre le Premier Consul Bonaparte, 14 Nivôse an 9, AN, F7 6271.

2. Karelle VINCENT, Le régicide, de Saint Réjant à Gorguloff (1800-1932).Perceptions et représentations, dir. J.-M. Berlière, Université de Bourgogne, 2003, 865 p. S'orientant résolument dans cette perspective (L'Ouragan homicide. L'attentat politique en France au XIX ${ }^{e}$ siècle, Seyssel, Champ Vallon, 2010, 352 p.), Karine SALOMÉ vient de proposer une relecture du 3 Nivôse, dans le cadre d'une analyse comparée des modes opératoires de la violence révolutionnaire et de leur réception («L'attentat de la rue Nicaise en 1800 : l'irruption d'une violence inédite? ", Revue d'histoire du XIX siècle, $\mathrm{n}^{\circ} 40,2010$, p. 59-75).

3. À l'instar de Jean LORÉDAN, La Machine infernale de la rue Nicaise, Paris, Perrin, "Drames judiciaires d'autrefois », 1924. 
4. Indiquons quelques jalons : Émile MARCO DE SAINT-HILAIRE, Histoire des conspirations et attentats contre le gouvernement et la personne de Napoléon, Paris, Fellens, 1847, Henri GAUBERT, Conspirateurs au temps de Napoléon I ${ }^{\text {er }}$, Paris, Flammarion, 1962; Louis DE VILLEFOSSE et Janine BOUISSOUNOUSE, L'Opposition à Napoléon, Paris, Flammarion, 1969, Gérard MINART, Les Opposants à Napoléon. L'élimination des royalistes et des républicains (1800-1815), Toulouse, Privat, 2003.

5. Dans cette optique, Natalie PETITEAu n'évoque la Machine infernale que pour revenir sur la signification politique de l'exécution de ses auteurs (Les Français et l'Empire, 1799-1815, Paris, Boutique de l'Histoire, 2008, p. 99).

6. On tirera profit de la manière dont Nathalie ALZAS envisage lapremière terreur blanche languedocienne comme une stratégie d'intimidation visant à masquer la faiblesse véritable du royalisme intransigeant («Rites de violence et fêtes royalistes dans l'Hérault ou comment s'affirmer majoritaire sous le Directoire », dans Christine Peyrard (dir.), Minorités politiques en Révolution, Aix-en-Provence, Publications de l'Université de Provence, 2007, p. 111- 127).

7. Sur les assassinats politiques dans l'Allemagne de Weimar: George Mosse, De la Grande Guerre au totalitarisme. La brutalisation des sociétés européennes, Paris, Hachette, 1999 [Fallen Soldiers: Reshaping the Memory of the World Wars, 1990], p. 181-206.

8. Roger DUPUY, Les Chouans, Paris, Hachette, 1997, p. 83.

9. Ibid., p. 136-137.

10. Gilles malandain, L'Introuvable complot. Attentat, enquête et rumeur dans la France de la Restauration, Paris, Éd. de l'EHESS, 2011.

11. On ne dispose toutefois que de la copie de ce rapport du 2 Nivôse, extrait de la correspondance du commandant la gendarmerie de Maine-et-Loire, AN, $\mathrm{F}^{7} 6271$.

12. «Avant le 3 Nivôse, les individus connus pour l'exagération de leurs systèmes politiques paraissaient généralement gais et triomphans, quelques meneurs surtout ont multiplié les courses [...] dès le 5 Nivôse la consternation s'était répandue sur les figures de ces mêmes individus. Ces rapprochements donnent lieu de croire que les conspirateurs de Paris avaient dans les départemens une correspondance active, que leur infâme complot était connu de ceux qu'ils savaient enclins à y participer au moins de cœur ", le préfet de la Sarthe au ministre de la Police générale, 27 Nivôse an 9, AN, $\mathrm{F}^{7} 6232^{\mathrm{A}}$.

13. Huon DE PENANSTER, Une Conspiration en l'an XI et en l'an XII, Paris, Plon, 1896.

14. Olivier BlANC, Les Espions de la Révolution et de l'Empire, Paris, Perrin, 1995, p. 225-235.

15. Mémoires de M. de Bourienne, ministre d'État, Paris, Ladvocat, 1829, V, p. 274.

16. Jean RIEUX, La Chouannerie sur les pas de Cadoudal, Paris, Artra, 1985, p. 245-246.

17. Correspondance entre le préfet de l'Ille-et-Vilaine et le ministre de la Police générale en Prairial an 9, AN, $\mathrm{F}^{7} 6233$ et 6235.

18. Ernest D'HAUTERIVE, La Contre-Police royaliste en 1800, Paris, Perrin, 1931.

19. Rapport de la préfecture de police du 11 Germinal an 9, dans F. Alphonse AULARD, Paris sous le Consulat, Paris, Cerf, 1904, II, p. 230.

20. Note pour le ministre de la Police générale, Brumaire an 12, dans comte de MonTGaillard, Notice abrégée sur la vie, le caractère et les crimes des principaux assassins aux gages de l'Angleterre, Qui sont aujourd'hui traduits devant le Tribunal de la Seine, Paris, impr. impériale, an 12.

21. Jean THIRY, La Machine infernale, Paris, Berger-Levraut, 1952, 313 p.

22. Arnaud MARTEL, Les Historiens fantaisistes: M. Thiers, Histoire du Consulat et de l'Empire. Deuxième partie, Paris, E. Dentu, 1885, p. IV.

23. Rapport du ministre de la Police générale aux Consuls sur les auteurs de l'attentat du 3 Nivôse, 11 Pluviôse an 9, AN, $\mathrm{F}^{7} 6271$; l'idée sera reprise dans ses Mémoires, Paris, Imp. nationale, 1993 [1824], p. 161-169. 
24. Michael J. sydenham, "The Crime of 3 Nivôse (24 December 1800) », dans John Francis Bosher (dir.), French government and society, 1500-1850. Essays in memory of Alfred Cobban, Londres, Athlone Press, 1973, p. 295-320.

25. Projets que l'on peut suivre au fil des Rapports officiels et complets fait au gouvernement par le préfet de police de Paris, sur la conspiration tramée depuis six mois, contre le premier Consul Bonaparte, et l'explosion de la machine infernale, Paris, Marchand, an 9. Cette compilation de rapports du 3 Fructidor an 8 au 10 Nivôse an 9 est donc publiée après le 3 Nivôse, alors que les jacobins faisaient encore figure d'auteurs désignés, ce qui invite donc à considérer cette source policière comme une pièce à charge, rétrospectivement établie. La liste n'est pas exhaustive : il aurait également été question d'un assassin déguisé en marbrier ou d'une tentative lors de l'inauguration du mémorial à Kléber et Desaix (Henri GAUBERT, Conspirateurs..., op. cit.).

26. Ernest D'HAUTERIVE, La Contre-Police royaliste... op. cit., p. 114.

27. Napoléon Bonaparte, Correspondance générale, publiée par la Fondation Napoléon, vol. III, Pacifications, 1800-1802, Paris, Fayard, 2006.

28. Ernest DAUDET, La Police et les chouans sous le Consulat et l'Empire, 1800-1815, Paris, Plon \& Nourrit, 1895.

29. Le commissaire du Directoire exécutif près les tribunaux de la Loire-inférieure à la gendarmerie nationale du ressort, Nantes, 9 Frimaire an 6, AN, F ${ }^{9} 324$.

30. Notice abrégée..., op. cit., p. 4-5.

31. L'administration centrale du département de la Mayenne au ministre de la Police générale, 22 Nivôse an 7, AN, $\mathrm{F}^{7} 7534$

32. Les administrateurs du département de l'Orne au ministre de la Guerre, 28 Nivôse an 8, SHD, $\mathrm{B}^{5} 65$.

33. Le commissaire du gouvernement près l'administration centrale de l'Orne au ministre de la Police générale, 28 Nivôse an $8, \mathrm{AN}, \mathrm{F}^{7} 6229$.

34. Rapport du ministre de la Police générale aux Consuls sur les auteurs de l'attentat du 3 Nivôse, 11 Pluviôse an 9, AN, $\mathrm{F}^{7} 6271$.

35. Notice abrégée..., op. cit., p. 8

36. Note de Desgrées, 12 Frimaire, AN, $F^{7} 6271$.

37. Tableau de la situation de Paris du 14 Nivôse, dans F. Alphonse AULARD, Paris..., op. cit., p. 114.

38. Réal, conseiller d'État spécialement chargé de l'instruction et de la suite de toutes les affaires relatives à la tranquillité et à la sûreté intérieures de la République au préfet de police, 14 Ventôse an 12, APP, Aa 291 n $^{\circ} 203$.

39. Notice abrégée..., op. cit., p. 47-50.

40. Mémoires de M. de Bourrienne...op. cit., IV, p. 309.

41. Avis d'un habitué du Palais, sur quelques points relatifs à la procédure contre la conspiration, p. 7, APP, Aa 299 n $^{\circ} 473$.

42. Notice abrégée..., op. cit., p. 67.

43. Procès-verbal du préfet de police, contenant examen par le citoyen Monge de la machine saisi sur Chevalier, 19 Brumaire an 9 dans Rapports officiels..., op. cit.

44. C'est là, sommairement résumée, la piste problématique explorée par Karine SALomé (art. cit).

45. Rapport du ministre de la Police générale aux Consuls sur les auteurs de l'attentat du 3 Nivôse, 11 Pluviôse an 9, AN, $\mathrm{F}^{7} 6271$.

46. Journal de Paris, 5 Floréal an 9.

47. Le commissaire du gouvernement près le tribunal de première instance de la Rochelle au ministre de la Justice, 2 juin $1804, \mathrm{AN}, \mathrm{BB}^{18} 479$.

48. Procès-verbal du juge de paix du canton de Caen, le 7 novembre 1800, sur l'arrestation de Mme Vaudabon à Bayeux par la gendarmerie, AN, $\mathrm{F}^{7} 6233$. 
49. L'article concomitant de Karine SALOMÉ s'attache particulièrement à cette dimension, art. cit., p. 63-67.

50. Compte que rend le préfet de police, au Ministre de l'Intérieur, de l'emploi des fonds provenans des dons de particuliers en faveur des Victimes de l'attentat du 3 Nivôse, an 9, Paris, J.-R. Lottin, an X.

51. Tableau de la situation de Paris du 10 Nivôse an 9, dans F. Alphonse AULARD, Paris..., op. cit., p. 106.

52. Un mot sur le procès de la conjuration, an 12, APP, Aa 299 p. 474.

53. Pour cerner la réalité de l'implication britannique : Elizabeth SPARROW, Secret Service. British agents in France, 1792-1815, Woodbridge-Rochester, Boydell Press, 1999, p. 217-223 et 267-297.

54. F. Alphonse AULARD, Paris..., op. cit., p. 101.

55. Journal de Paris, 7 Nivôse an 9.

56. Ibid., 4 Messidor an 9.

57. Témoignages historiques ou Quinze ans de haute police sous Napoléon, par M. Desmarest, chef de cette partie pendant tout le Consulat et l'Empire, Paris, Levavasseur-Bousquet, 1833, p. 29.

58. Claude LANGLoIS, «Complots, propagandes et répression policière en Bretagne sous l'Empire (1806-1807) », Annales de Bretagne, LXXVIII-2, juin 1971, p. 369-421.

59. Bulletin de police du 10 août 1807, dans Ernest d'HAUTERIVE (éd.), La Police secrète du Premier Empire. Bulletins quotidiens adressés par Fouché à l'Empereur, Paris, Perrin-Clavreuil, 1922, III.

\section{RÉSUMÉS}

En mettant fin aux opérations militaires, la pacification de l'hiver 1800 disqualifie par le fait même en actes de brigandage les violences commises par les chouans insoumis. Lecture sommaire et tendancieuse sans doute mais qui a été avalisée par la configuration historiographique: les spécialistes de la police napoléonienne prennent alors le relais des historiens de la guerre civile. Un tel compartimentage, redoublé par la scission opérée entre Paris et l'Ouest, altère la compréhension de la chouannerie en créant un hiatus dans ses modes d'action. C'est en effet masquer une continuité réelle entre les attentats contre Bonaparte et l'usage délibéré de la terreur dans les départements de l'Ouest par des bandes s'employant à déstabiliser l'ordre républicain et à saboter le processus de réconciliation au moyen d'assassinats publics et d'enlèvements spectaculaires lors des périodes dites d'entre-chouannage ou de chouannerie à volonté. Mettre en évidence cette stratégie d'intimidation éclaire d'un jour nouveau l'affaire de la Machine infernale, rue Nicaise le 3 Nivôse an 9, et la conspiration de l'an 12: pour s'en convaincre, il n'est qu'à envisager l'itinéraire de leurs auteurs, qui sauront toutefois emprunter in fine le mode opératoire projeté par les jacobins.

By ending the military operations, the pacification of the winter 1800 disqualifies in acts of robbery the violence committed by the rebellious Chouans. Not doubt it is a ummary and biased reading but it was endorsed by the historiographical configuration: specialists of the Napoleonic police relaying historians of the civil war. Such a compartmentalization, doubled by the split operated between Paris and the West, alters the understanding of the revolt of the Chouans by creating a hiatus in its modes of action. It is indeed to mask a real continuity between attempts against Bonaparte and the deliberated use of terror in the western departments intending to destabilize the republican order and to sabotage the process of reconciliation by means of public murders and spectacular kidnappings during the so-called periods of "chouannerie à volonté" 
(at will). To remind this strategy of intimidation sheds new light on two famous affairs: the "Infernal Machine" of the Saint-Nicaise street and the Cadoudal conspiracy.

INDEX

Mots-clés : Cadoudal, chouannerie, contre-terrorisme, machine infernale, violence politique Keywords : Cadoudal, chouannerie, counter-terrorism, machine infernale, political violence

\section{AUTEUR}

\section{AURÉLIEN LIGNEREUX}

MCF à l'Université d'Angers - rattaché au CERHIO (UMR 6258)

aurelien.lignereux@univ-angers.fr 